\title{
Recent Total Ionizing Dose and Displacement Damage Compendium of Candidate Electronics for NASA Space Systems
}

\author{
Donna J. Cochran', Alvin J. Boutte ${ }^{2}$, Michael J. Campola ${ }^{2}$, Martin A. Carts ${ }^{2}$, Megan C. Casey ${ }^{2}$, Dakai Chen², \\ Kenneth A. LaBel ${ }^{2}$, Raymond L. Ladbury', Jean-Marie Lauenstein ${ }^{2}$, Cheryl J. Marshall ${ }^{2}$, \\ Martha V. O'Bryan', Timothy R. Oldham³, Jonathan A. Pellish' ${ }^{2}$. Anthony B. Sanders', and Michael A. Xapsos ${ }^{2}$ \\ 1. MEI Technologies, Inc., Seabrook, MD 20706 USA \\ 2. NASA Goddard Space Flight Center (GSFC), Code 561.4, Greenbelt, MD 20771 USA \\ 3. Dell Perot Systems Inc., Fairfax, VA 22031 USA
}

\begin{abstract}
Vulnerability of a variety of candidate spacecraft electronics to total ionizing dose and displacement damage is studied. Devices tested include optoelectronics, digital, analog, linear bipolar devices, and hybrid devices.

Index Terms- Displacement Damage, Optoelectronics, Proton Damage, Single Event Effects, and Total Ionizing Dose.
\end{abstract}

\section{INTRODUCTION}

NASA spacecraft are subjected to a harsh space environment that includes exposure to various types of ionizing radiation. Long-term exposure to radiation has been known to affect the function of the spacecraft electronics. As a result flight parts must be tolerant to radiation-induced Total Ionizing Dose (TID) and displacement damage (DD) effects for space approval or parts must be mitigated by shielding or other methods to reduce TID effects. Hence, the effects of TID and proton DD need to be evaluated by test in order to determine risk to space projects.

The test results presented here were gathered to establish the sensitivity of candidate spacecraft electronics to TID and/or proton damage. For similar results on single event effects (SEE), a companion paper has also been submitted to the 2011 IEEE NSREC Radiation Effects Data Workshop entitled: "Recent Single Event Effects Compendium of

This work was supported in part by the NASA Electronic Parts and Packaging Program (NEPP), NASA Flight Projects, and the Defense Threat Reduction Agency (DTRA) under LACRO\# 10-4977I and 11-4395I.

Donna J. Cochran, MEI Technologies Inc., work performed for NASA Goddard Space Flight Center, Code 561.4, Greenbelt, MD 20771 (USA), phone: 301-286-8258, fax: 301-286-4699, email: Donna.J.Cochran@nasa.gov.

Jonathan A. Pellish, NASA/GSFC, Code 561.4, Greenbelt, MD 20771 (USA), phone: 301-286-6523, email: Jonathan.A.Pellish@nasa.gov.

Megan C. Casey, NASA/GSFC, Code 561.4, Greenbelt, MD 20771 (USA), phone: 301-286-1151, email: Megan.C.Casey@nasa.gov.

Raymond L. Ladbury, NASA/GSFC, Code 561.4, Greenbelt, MD 20771 (USA), phone: 301-286-1030, email: Raymond.L.Ladbury@nasa.gov.

Timothy R. Oldham, Dell Perot Systems Government Services, Inc., work performed for NASA Goddard Space Flight Center, Code 561.4, Greenbelt, MD 20771 (USA), phone: 301-286-5489, email: Timothy.R.Oldham@nasa.gov.

Martha V. O'Bryan is with MEI Technologies Inc., work performed for NASA Goddard Space Flight Center, Code 561.4, Greenbelt, MD 20771 (USA), phone: 301-286-1312, email: Martha.V.Obryan@nasa.gov.

Alvin Boutte, Michael J. Campola, Martin A. Carts, Dakai Chen, Kenneth A. LaBel, Cheryl J. Marshall, Jean-Marie Lauenstein, Anthony B. Sanders, and Michael Xapsos are with NASA/GSFC, Code 561.4, Greenbelt, MD 20771 (USA), phone:301-286-8046 (Sanders), 301-286-9936 (LaBel), email: Anthony.B.Sanders@nasa.gov, Kenneth.A.LaBel@nasa.gov.
Candidate Electronics for NASA Space Systems" by M. O'Bryan, et al. [1]

\section{Test TeChNiQues and Setup}

Unless otherwise noted, all tests were performed at room temperature and with nominal power supply voltages.

\section{A. Test Methods - TID}

TID testing was performed using a Co-60 source. Dose rates used for testing were between 0.0005 and $50 \mathrm{rads}(\mathrm{Si}) / \mathrm{s}$.

\section{B. Test Methods - Proton}

Proton DD/TID tests were performed at the University of California at Davis - Crocker Nuclear Laboratory (UCD$\mathrm{CNL}$ ) using a 76" cyclotron (maximum energy of $63 \mathrm{MeV}$ ) and the Lawrence Berkeley National Laboratory (LBNL) Berkeley Accelerator Space Effects (BASE). Table I lists the proton damage test facilities and energies used on the devices.

TABLE I

PROTON TEST FACILITIES

\begin{tabular}{|l|c|}
\hline \multicolumn{1}{|c|}{ Facility } & $\begin{array}{c}\text { Proton Energy, } \\
(\mathrm{MeV})\end{array}$ \\
\hline $\begin{array}{l}\text { University of California at Davis - } \\
\text { Crocker Nuclear Laboratory (UCD-CNL) }\end{array}$ & $6.5-63$ \\
\hline $\begin{array}{l}\text { Lawrence Berkeley National Laboratory } \\
\text { (LBNL) Berkeley Accelerator Space Effects } \\
\text { (BASE) }\end{array}$ & 50 \\
\hline
\end{tabular}

\section{Test Methods-Electron}

Electron DD/TID tests were performed at Rensselaer Polytechnic Institute's (RPI) Gaerttner Linear Accelerator Laboratory. The electron energies used were between 5 and $50 \mathrm{MeV}$.

\section{Test Results Overview}

Abbreviations for principal investigators (PIs) are listed in Table II. Abbreviations and conventions are listed in Table III. Please note that these test results can depend on operational conditions. Complete test reports are available online at http://radhome.gsfc.nasa.gov [3].

To be presented by Alvin Boutte at the International Electrical and Electronics Engineering (IEEE) Nuclear and Space Radiation Effects Conference (NSREC), July 27, 2011, Las Vegas, NV, and published on nepp.nasa.gov and radhome.gsfc.nasa.gov. 
TABLE II

LIST OF PRINCIPAL INVESTIGATORS

\begin{tabular}{|c|c|}
\hline Abbreviation & Principal Investigator (PI) \\
\hline MiC & Michael Campola \\
\hline $\mathrm{MaC}$ & Martin Carts \\
\hline $\mathrm{MeC}$ & Megan Casey \\
\hline $\mathrm{DC}$ & Dakai Chen \\
\hline $\mathrm{RL}$ & Raymond Ladbury \\
\hline $\mathrm{JML}$ & Jean-Marie Lauenstein \\
\hline $\mathrm{CM}$ & Cheryl Marshall \\
\hline $\mathrm{TO}$ & Timothy Oldham \\
\hline JP & Jonathan Pellish \\
\hline $\mathrm{AS}$ & Anthony (Tony) Sanders \\
\hline $\mathrm{MX}$ & Michael Xapsos \\
\hline
\end{tabular}

TABLE III

ABBREVIATIONS AND CONVENTIONS

\begin{tabular}{|c|c|}
\hline ACRONYM/ DEFINITION & ACRONYM/ DEFINITION \\
\hline 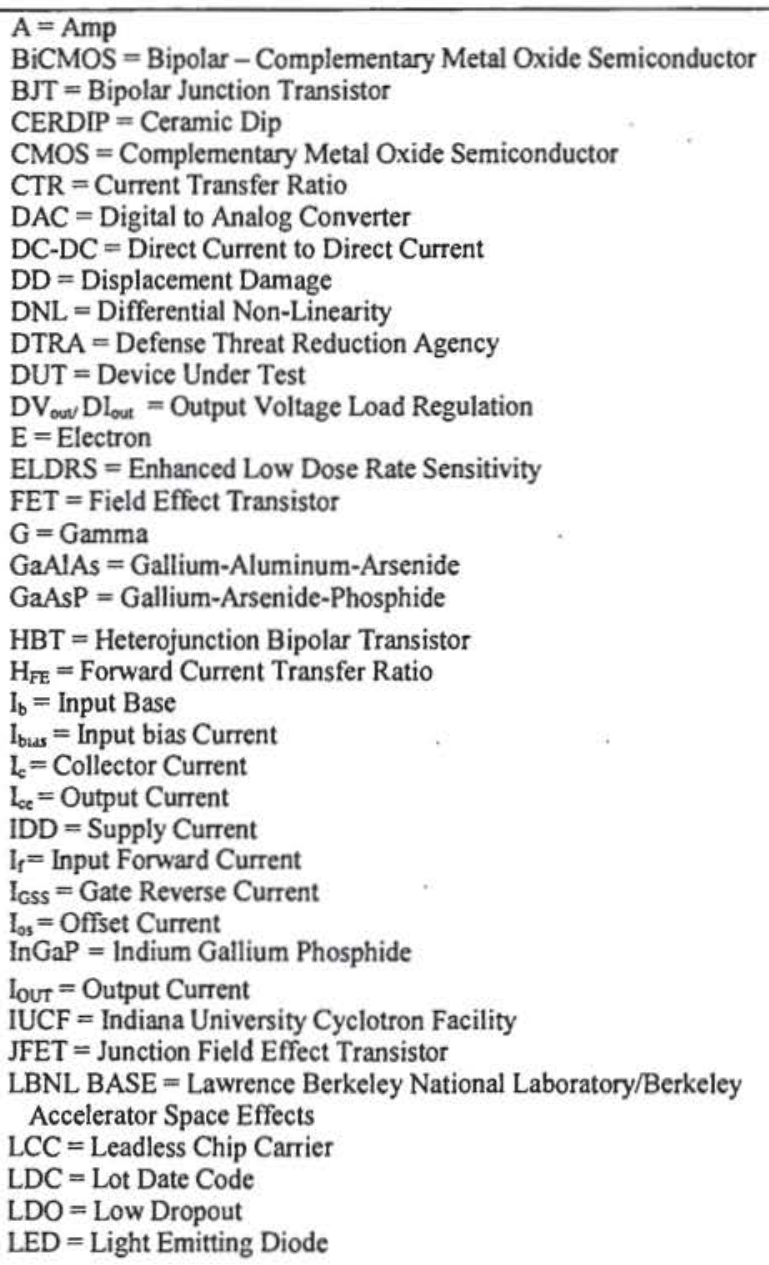 & $\begin{array}{l}\text { LDR }=\text { Low Dose Rate } \\
\text { MeV }=\text { Mega Electron Volt } \\
\mathrm{mA}=\text { milliamp } \\
\text { MOSFET }=\text { Metal Oxide Semiconductor Field Effect } \\
\mathrm{N} / \mathrm{A}=\text { Not Available } \\
\text { Op-Amp }=\text { Operational Amplifier } \\
\mathrm{P}=\text { Proton } \\
\mathrm{PI}=\text { Principal Investigator } \\
\mathrm{REAG}=\text { Radiation Effects \& Analysis Group } \\
\mathrm{RPI}=\text { Rensselaer Polytechnic Institute Gaerttner Linear Accelerator } \\
\text { Laboratory } \\
\text { SEE }- \text { Single Event Effects } \\
\text { Spec }=\text { Specification(s) } \\
\text { TID }=\text { Total lonizing Dose } \\
\text { UCD-CNL = University of California at Davis - Crocker Nuclear Laboratory } \\
\mathrm{V}_{\mathrm{bus}}=\text { Bias Voltage } \\
\mathrm{V}_{\mathrm{ce}}=\text { Collector Emitter Voltage } \\
\mathrm{V}_{\mathrm{cEst}}=\text { Collector }- \text { Emitter Saturation Voltage } \\
\mathrm{V}_{\mathrm{fH}}=\text { High Level Input Voltage } \\
\mathrm{V}_{\mathrm{in}}=\text { Voltage In } \\
\mathrm{V}_{\mathrm{os}}=\text { Offset Voltage } \\
\mathrm{V}_{\text {out }}=\text { Output Voltage } \\
\mathrm{V}_{\text {ret }}=\text { Reference Voltage } \\
\mathrm{V}_{\mathrm{tb}}=\text { Threshold Voltage } \\
\mathrm{V}_{\mathrm{z}}=\text { Reverse Breakdown Voltage }\end{array}$ \\
\hline
\end{tabular}

To be presented by Alvin Boutte at the International Electrical and Electronics Engineering (IEEE) Nuclear and Space Radiation Effects Conference (NSREC), July 27, 2011, Las Vegas, NV, and published on nepp.nasa.gov and radhome.gsfc.nasa.gov. 
TABLE IV

SUMMARY OF TID AND DD TEST RESULTS

\begin{tabular}{|c|c|c|c|c|c|c|c|c|}
\hline Part Number & Manufacturer & LDC & $\begin{array}{l}\text { Technology/ } \\
\text { Device Function }\end{array}$ & PI & Summary of Results & 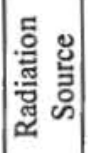 & 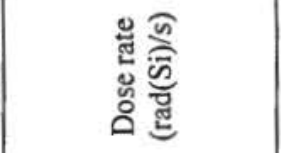 & 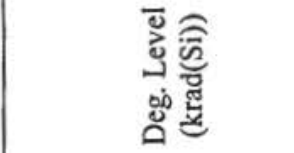 \\
\hline \multicolumn{9}{|c|}{ Analog Digital Converter/Digital Analog Converter } \\
\hline AD5544ARS & $\begin{array}{l}\text { Analog } \\
\text { Devices }\end{array}$ & $\begin{array}{c}0332,0409 \\
0827, \& 1028\end{array}$ & $\begin{array}{l}\text { CMOS / Quad 16-bit } \\
\text { DAC (0827 \& 1028); } \\
\text { BiCMOS/Quad 16-bit } \\
\text { DAC (0332 \& 0409) }\end{array}$ & JP & $\begin{array}{l}\text { 0827: Functional failure was observed in one } \\
\text { DUT at } 2 \text { krad(Si). } 1028 \text { : Functional failure } \\
\text { observed in one DUT at } 10 \text { krad(Si). Both } \\
0827 \text { \& } 1028 \text { show TID sensitivities to IDD, } \\
\text { output leakage, \& DNL errors - biased } \\
\text { irradiation is worst case. } 0332 \text { \& } 0409 \text { passed } \\
\text { all tests through } 15 \mathrm{krad}(\mathrm{Si}) \text { at which point } \\
\text { the biased samples' IDD went out of spec. } \\
\text { Both date codes showed no functional } \\
\text { failures through } 20 \mathrm{krad}(\mathrm{Si}) \text {. }\end{array}$ & G & $\mid \begin{array}{c}0.01(0827), 2 \\
(1028), 0.01(0332 \& \\
0409)\end{array}$ & $\begin{array}{c}0 \leq x \leq 2(0827) ; 7 \leq x \\
\leq 10(1028) ; 10 \leq x \leq \\
15(0332 \& 0409)\end{array}$ \\
\hline AD585 & $\begin{array}{l}\text { Analog } \\
\text { Devices }\end{array}$ & 8440 & \begin{tabular}{|c|} 
Bipolar / \\
Sample \& Hold Amp
\end{tabular} & MX & $\begin{array}{l}\text { Droop rate out of spec at } 15 \mathrm{krad} ; \mathrm{V}_{\text {os }} \text { at } 40 \\
\mathrm{krad}(\mathrm{Si}) \text {. }\end{array}$ & G & 0.01 & $\begin{array}{c}10<\text { droop rate }<15 \\
30<\text { offset } \mathrm{V}<40\end{array}$ \\
\hline \multicolumn{9}{|l|}{ Flash } \\
\hline MT29F8G0AAAWP & Micron & 0948 & $\begin{array}{l}\text { CMOS/8G NAND } \\
\text { Flash } \\
\end{array}$ & TO & $\begin{array}{l}50 \mathrm{krad}(\mathrm{Si}) .<\text { Fail }<75 \mathrm{krad}(\mathrm{Si}) ; \text { loss of } \\
\text { erase/write function. }\end{array}$ & G & 50 & 75 \\
\hline K9F8G08U0M & Samsung & 1031 & $\begin{array}{l}\text { CMOS/8G NAND } \\
\text { Flash }\end{array}$ & TO & $\begin{array}{l}400 \mathrm{krad}(\mathrm{Si})<\text { Fail }<500 \mathrm{krad}(\mathrm{Si}) ; \text { partial loss } \\
\text { of Erase/Write functions. }\end{array}$ & G & 50 & 500 \\
\hline \multicolumn{9}{|l|}{ Miscellaneous } \\
\hline $\begin{array}{l}\text { LM139AWRQMLV } \\
\text { (14-lead CERDIP) }\end{array}$ & Natl Semi & JM046X13 & $\begin{array}{l}\text { Bipolar / Analog } \\
\text { Comparator }\end{array}$ & DC & $\begin{array}{l}\text { Parameters within spec after } 10 \mathrm{krad}(\mathrm{Si}) \text { for } \\
\text { devices irradiated at } 0.5 \mathrm{mrad}(\mathrm{Si}) / \mathrm{s} \text {. }\end{array}$ & G & 0.0005 & $>10$ \\
\hline HS-1840ARH & Intersil & X0902ABB8 & $\begin{array}{l}\text { CMOS/ Analog } \\
\text { Multiplexer }\end{array}$ & MX & $\begin{array}{l}\text { Outputs \& reference voltage held at } \\
\text { application specific }+3.3 \mathrm{~V} \text {. }\end{array}$ & G & 16.7 & $>100$ \\
\hline Ram - 6+ & Mini-Circuits & N/A & $\begin{array}{c}\text { Bipolar / } \\
\text { RF Amplifier }\end{array}$ & MX & $\begin{array}{l}\text { For } 2 \text { lots of } 8 \text { parts each worst-case gain } \\
\text { degradation at } 50 \mathrm{krad} \text { was } 15 \% \text {. }\end{array}$ & G & 0.02 & $>50$ \\
\hline ISL74422ARH/1SL4422BRH & Intersil & 0948 & $\begin{array}{c}\text { BiCMOS/ } \\
\text { MOSFET Driver }\end{array}$ & RL & $\begin{array}{l}\text { Minor degradation. All parts within spec to } \\
>100 \mathrm{krad}(\mathrm{Si}) \text {. }\end{array}$ & G & 0.01 & $>100$ \\
\hline MCM2760-4M & QTech & 0135 & $\begin{array}{l}\text { Hybrid / Crystal } \\
\text { Oscillator }\end{array}$ & $\mathrm{MeC}$ & $\begin{array}{l}\text { All parameters stayed within spec limits to } 3 \\
\text { Mrad(Si). }\end{array}$ & G & 74 & $>3000$ \\
\hline \multicolumn{9}{|c|}{ Operational Amplifier/Amplifier } \\
\hline \multirow{4}{*}{$\begin{array}{l}\text { LM158AJRLQMLV } \\
\text { (8-lead CERDIP) }\end{array}$} & \multirow{4}{*}{ Natl Semi } & \multirow{4}{*}{ 7W4453G019 } & \multirow{4}{*}{$\begin{array}{l}\text { Bipolar/ } \\
\text { Op-Amp }\end{array}$} & \multirow{4}{*}{ DC } & \multirow{4}{*}{$\begin{array}{l}\text { Parameters within spec after } 100,70,50, \& \\
30 \mathrm{krad}(\mathrm{Si}) \text { for devices irradiated. at } 10,5,1 \text {, } \\
\& 0.5 \mathrm{mrad}(\mathrm{Si}) \text {, with the exception of } 1 \text { part } \\
\text { at } 5 \mathrm{mrad}(\mathrm{Si}) / \mathrm{s} \text {, which parametrically failed } \\
\text { after } 70 \mathrm{krad}(\mathrm{Si}) .5 \mathrm{mrad}(\mathrm{Si}) / \mathrm{s}(1 \text { part): } 60< \\
\mathrm{I}_{\mathrm{b}}<70 \mathrm{krad}(\mathrm{Si}) . \text { Devices Exhibit dose rate } \\
\text { enhancement after } 50 \mathrm{krad}(\mathrm{Si}) \text {. }\end{array}$} & \multirow{4}{*}{ G } & 0.01 & $>100$ \\
\hline & & & & & & & 0.0005 & $60<\mathrm{I}_{b}<70$ \\
\hline & & & & & & & 0.001 & $>50$ \\
\hline & & & & & & & 0.0005 & $>30$ \\
\hline
\end{tabular}

To be presented by Alvin Boutte at the International Electrical and Electronics Engineering (IEEE) Nuclear and Space Radiation Effects Conference (NSREC), July 27, 2011, Las Vegas, NV, and published on nepp.nasa.gov and radhome.gsfc.nasa.gov. 


\begin{tabular}{|c|c|c|c|c|c|c|c|c|}
\hline Part Number & Manufacturer & LDC & $\begin{array}{l}\text { Technology/ } \\
\text { Device Function }\end{array}$ & PI & Summary of Results & 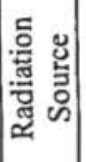 & 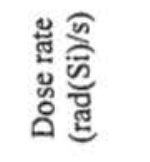 & 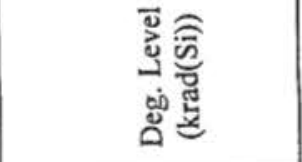 \\
\hline \multirow{2}{*}{$\begin{array}{l}\text { LMI24AJRQMLV } \\
\text { (14-lead CERDIP) }\end{array}$} & \multirow{2}{*}{ Natl Semi } & \multirow{2}{*}{ 9R5469G019 } & \multirow{2}{*}{$\begin{array}{l}\text { Bipolar/ } \\
\text { Op-Amp }\end{array}$} & \multirow{2}{*}{ DC } & \multirow{2}{*}{$\begin{array}{l}\text { Exhibits no dose rate enhancement. } \\
\text { Parameters within spec after } 10 \& 13 \\
\text { krad(Si) for devices irradiated at } 10 \& 0.5 \\
\mathrm{mrad}(\mathrm{Si}) / \mathrm{s} \text {. }\end{array}$} & \multirow[b]{2}{*}{ G } & 0.01 & $>13$ \\
\hline & & & & & & & 0.0005 & $>10$ \\
\hline \multirow{3}{*}{$\begin{array}{l}\text { RH1013MH } \\
\text { (TO5 metal can) }\end{array}$} & \multirow{3}{*}{$\begin{array}{c}\text { Linear } \\
\text { Technology }\end{array}$} & \multirow{3}{*}{$0329 \mathrm{~A} / 9513 \mathrm{~A}$} & \multirow{3}{*}{$\begin{array}{l}\text { Bipolarl } \\
\text { Dual Precision } \\
\text { Op-Amp }\end{array}$} & \multirow{3}{*}{ DC } & \multirow{3}{*}{$\begin{array}{l}\text { Parameters within spec after } 100,20, \& 10 \\
\operatorname{krad}(\mathrm{Si}) \text { for } 5,1, \& 0.5 \mathrm{mrad}(\mathrm{Si}) / \mathrm{s} \text {. }\end{array}$} & \multirow{3}{*}{ G } & 0.005 & $>100$ \\
\hline & & & & & & & 0.001 & $>20$ \\
\hline & & & & & & & 0.0005 & $>10$ \\
\hline \multirow{3}{*}{$\begin{array}{l}\text { RH1013MJ8 } \\
\text { (CERDIP) }\end{array}$} & \multirow{3}{*}{$\begin{array}{c}\text { Linear } \\
\text { Technology }\end{array}$} & \multirow{3}{*}{$0305 \mathrm{~A} / 0337 \mathrm{~A}$} & \multirow{3}{*}{$\begin{array}{l}\text { Bipolar/ } \\
\text { Op-Amp }\end{array}$} & \multirow{3}{*}{ DC } & \multirow{3}{*}{$\begin{array}{l}\text { Parameters within spec after } 100,20, \& 10 \\
\operatorname{krad}(\mathrm{Si}) \text { for } 5,1, \& 0.5 \mathrm{mrad}(\mathrm{Si}) / \mathrm{s} \text {. }\end{array}$} & \multirow{3}{*}{ G } & 0.005 & $>100$ \\
\hline & & & & & & & 0.001 & $>20$ \\
\hline & & & & & & & 0.0005 & $>10$ \\
\hline RH310 (Ceramic Flat-8) & $\begin{array}{c}\text { ST } \\
\text { Micro- } \\
\text { electronics }\end{array}$ & $30849 A$ & $\begin{array}{l}\text { Bipolar/ } \\
\text { Op-Amp }\end{array}$ & DC & $\begin{array}{l}\text { Parameters within spec for parts irradiated at } \\
5 \mathrm{mrad}(\mathrm{Si}) / \mathrm{s} \text { after } 5 \mathrm{krad}(\mathrm{Si}) .1 \mathrm{mrad}(\mathrm{Si}) / \mathrm{s} \\
\text { irradiation currently in progress. }\end{array}$ & G & 0.0005 & $>5$ \\
\hline OP497BRC & $\begin{array}{l}\text { Analog } \\
\text { Devices }\end{array}$ & 0946A & $\begin{array}{l}\text { Precision Picoampere } \\
\text { Input Current Quad/ } \\
\text { Op-Amp }\end{array}$ & JP & \begin{tabular}{|l|} 
Significant degradation of all measured \\
parameters after $10 \mathrm{krad}(\mathrm{Si}) . \mathrm{I}_{\text {bias }} \& \mathrm{l}_{\mathrm{os}} \&$ \\
voltage parametric means out of spec by 4 \\
krad(Si), but may be tolerable in a given \\
application. Co-60 low dose rate testing only.
\end{tabular} & G & 0.01 & $2.5 \leq x \leq 5$ \\
\hline AD648 & $\begin{array}{l}\text { Analog } \\
\text { Devices }\end{array}$ & 9643 & $\begin{array}{l}\text { Bipolar/ } \\
\text { Op-Amp }\end{array}$ & $\mathrm{MeC}$ & $\begin{array}{l}\text { The spec for } I_{\text {bias }} \& I_{\text {os }} \text { was exceeded by one } \\
\text { biased part at } 2 \mathrm{krad}(\mathrm{Si}) \text {. }\end{array}$ & G & 0.01 & $0<\mathrm{I}_{\mathrm{os}}<2,0<\mathrm{I}_{\text {bias }}<2$ \\
\hline PA10 & Apex & 0936 & $\begin{array}{l}\text { Bipolar / } \\
\text { Op-Amp }\end{array}$ & $\mathrm{MiC}$ & $\begin{array}{l}\text { All devices stayed within spec up to } 50 \\
\text { krad(Si). }\end{array}$ & G & 0.01 & $x>50$ \\
\hline RHI056A & $\begin{array}{c}\text { Linear } \\
\text { Technology }\end{array}$ & $0921 \mathrm{~A}$ & \begin{tabular}{|c|} 
Bipolar/ \\
JFET Input Op-Amp \\
\end{tabular} & MX & 4 parts tested all within specs at $50 \mathrm{krad}(\mathrm{Si})$. & G & 0.02 & $>50$ \\
\hline AD524 & $\begin{array}{l}\text { Analog } \\
\text { Devices }\end{array}$ & 0939A & \begin{tabular}{|c|} 
Bipolar / \\
Instrumentation Amp \\
\end{tabular} & MX & $\mathrm{I}_{\text {bias }}$ out of specs at $10 \mathrm{krad}(\mathrm{Si})$. & G & 0.02 & $5<\mathrm{I}_{\text {bias }} \mathrm{I}<10$ \\
\hline OP400 & $\begin{array}{l}\text { Analog } \\
\text { Devices }\end{array}$ & 0204 & $\begin{array}{c}\text { Bipolar/ } \\
\text { Quad Op-Amp }\end{array}$ & RL & $\begin{array}{l}\mathrm{I}_{6} \text { is most sensitive parameter; } \mathrm{V}_{\mathrm{IO}} \text { second; } \\
\text { all other parameters in specification to }>60 \\
\mathrm{krad}(\mathrm{Si}) \text {. }\end{array}$ & G & 0.01 & $9<\mathrm{l}_{b}<11.5,15<\mathrm{V}_{10}<20$ \\
\hline \multicolumn{9}{|c|}{ Power Mosfet/Misc. Power/DC-DC } \\
\hline SiB455EDK & Vishay & BKW 9QZ & $\begin{array}{l}\text { Power p-type } \\
\text { TrenchFET }\end{array}$ & JML & $\begin{array}{l}V_{\text {th }} \text { degrades with TID. Parameters remained } \\
\text { within spec. }\end{array}$ & G & $4-17$ & $>150$ \\
\hline SPT6235MS & SSDI & 0624 & $\begin{array}{c}\text { General Purpose High } \\
\text { Voltage/Power NPN } \\
\text { BJT }\end{array}$ & JP & $\begin{array}{l}\text { Tested with } \mathrm{l}_{\mathrm{c}} \text { of } 0.25 \& 0.51 \mathrm{~A}, \mathrm{~V}_{\mathrm{b}}=5 \mathrm{~V}, \& \\
\mathrm{~V}_{\mathrm{ce}}=155.6 \mathrm{~V} \text {. Mean } \mathrm{h}_{\mathrm{FE}} \text { stayed within } \\
\text { application spec for the duration of the test. }\end{array}$ & G & $2-20$ & $>2 \times 10^{6}$ \\
\hline LS2805 & $\begin{array}{c}\text { Intl } \\
\text { Rectifier }\end{array}$ & 0536 & $\begin{array}{c}\text { Hybrid /High } \\
\text { Reliability Radiation } \\
\text { Hardened DC/DC } \\
\text { Converter }\end{array}$ & $\mathrm{MeC}$ & $\begin{array}{l}\mathrm{V}_{\text {out }} \text { decreased below the specified limit } \\
\text { between } 100 \& 300 \mathrm{krad}(\mathrm{Si}) \text { for one part. }\end{array}$ & G & 67.6 & $100<V_{\text {out }}<300$ \\
\hline
\end{tabular}

To be presented by Alvin Boutte at the International Electrical and Electronics Engineering (IEEE) Nuclear and Space Radiation Effects Conference (NSREC), July 27, 2011, Las Vegas, NV, and published on nepp.nasa.gov and radhome.gsfc.nasa.gov. 


\begin{tabular}{|c|c|c|c|c|c|c|c|c|}
\hline Part Number & Manufacturer & LDC & $\begin{array}{l}\text { Technology/ } \\
\text { Device Function }\end{array}$ & PI & Summary of Results & 总 : & 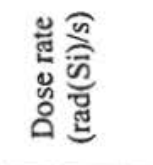 & 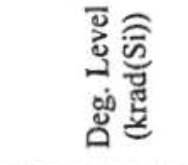 \\
\hline \multicolumn{9}{|c|}{ Transistors/BJTs/FETs } \\
\hline JANS2N3866 & Semicoa & 0721 & $\begin{array}{l}\text { General Purpose } \\
\text { NPN BJT }\end{array}$ & JP & $\begin{array}{l}\text { Tested with } \mathrm{I}_{\mathrm{c}} \text { of } 0.25 \& 0.51 \mathrm{~A} \text { with a pulsed } \\
\text { base current \& } \mathrm{V}_{\mathrm{ce}}=4.4 \mathrm{~V} \text {. Mean } \mathrm{h}_{\mathrm{FE}} \text { stayed } \\
\text { within application spec for the duration of } \\
\text { the test. }\end{array}$ & G & $2-20$ & $>2 \times 10^{6}$ \\
\hline 2N5116 & Vishay & S0618 & $\begin{array}{l}\text { JFET / } \\
\text { P-Channel Analog } \\
\text { Switch } \\
\end{array}$ & $\mathrm{MeC}$ & $\begin{array}{l}\mathrm{I}_{\text {GSs }} \text { exceeded the specified limits at the first } \\
\text { datapoint of } 10 \mathrm{krad}(\mathrm{Si}) \text {. }\end{array}$ & G & 31.3 & $0<\mathrm{I}_{\mathrm{GSS}}<10$ \\
\hline $2 \mathrm{~N} 2222 \mathrm{~A}$ & SSDI & 0686 & $\begin{array}{c}\text { Bipolar/ } \\
\text { Discrete Transistors } \\
\end{array}$ & MX & $D C h_{\mathrm{FE}}$ out of specs at $40 \mathrm{krad}(\mathrm{Si})$. & G & 16.7 & $30<\mathrm{h}_{\mathrm{FE}}<40$ \\
\hline 2N2907A & Semicoa & 0807 & $\begin{array}{c}\text { Bipolar/Switching \& } \\
\text { Small Signal } \\
\text { Transistors } \\
\end{array}$ & $\mathrm{MX}$ & DC current gain out of specs at $40 \mathrm{krad}(\mathrm{Si})$. & G & 16.7 & $30<\mathrm{h}_{\mathrm{FE}}<40$ \\
\hline 2N2907 & Micro Semi & 0513 & $\begin{array}{l}\text { Bipolar / PNP Silicon } \\
\text { Switching Transistor }\end{array}$ & $\mathrm{MeC}$ & $\begin{array}{l}h_{\mathrm{FE}} \text { for a } \mathrm{I}_{\mathrm{C}} \text { of } 0.1 \mathrm{~mA} \text { exceeded the spec } \\
\text { between } 30 \text { \& } 50 \mathrm{krad}(\mathrm{Si}) \text {. }\end{array}$ & G & 95 & $30<\mathrm{h}_{\mathrm{FE}}<50$ \\
\hline \multicolumn{9}{|c|}{ Voltage Reference/Voltage Regulator } \\
\hline \multirow{3}{*}{ LT1009IDR } & \multirow{3}{*}{$\begin{array}{c}\text { Texas } \\
\text { Instruments }\end{array}$} & \multirow{3}{*}{0606} & \multirow{3}{*}{$\begin{array}{l}\text { Bipolar/ } \\
\text { 2.5V Internal } \\
\text { Reference }\end{array}$} & \multirow{3}{*}{ DC } & \multirow{3}{*}{$\begin{array}{l}\text { Exhibits no dose rate enhancement. } \\
\text { Parameters within spec after } 100,30, \& 15 \\
\text { krad(Si) for the } 5,1, \& 0.5 \text { mrad(Si) parts. }\end{array}$} & \multirow{3}{*}{ G } & 0.005 & $>100$ \\
\hline & & & & & & & 0.001 & $>30$ \\
\hline & & & & & & & 0.0005 & $>15$ \\
\hline \multirow{3}{*}{ LM317KTTR } & \multirow{3}{*}{$\begin{array}{c}\text { Texas } \\
\text { Instruments }\end{array}$} & \multirow{3}{*}{0608} & \multirow{3}{*}{$\begin{array}{l}\text { Bipolar/ Positive } \\
\text { Voltage Regulator } \\
\text { 3-terminal }\end{array}$} & \multirow{3}{*}{ DC } & \multirow{3}{*}{$\begin{array}{l}\text { Parameters within spec after } 80,20, \& 15 \\
\text { krad(Si) for the } 5,1, \& 0.5 \mathrm{mrad}(\mathrm{Si}) \text { parts. } \\
\text { LDR enhancement observed for parts } \\
\text { irradiated at } 0.5 \text { \& } 1 \mathrm{mrad}(\mathrm{Si}) / \mathrm{s} \text { after } 20 \\
\text { krad(Si). }\end{array}$} & \multirow{3}{*}{ G } & 0.005 & $>80$ \\
\hline & & & & & & & 0.001 & $>20$ \\
\hline & & & & & & & 0.0005 & $>15$ \\
\hline \multirow{4}{*}{ TL750L05CDR } & \multirow{4}{*}{$\begin{array}{c}\text { Texas } \\
\text { Instruments }\end{array}$} & \multirow{4}{*}{0605} & \multirow{4}{*}{$\begin{array}{c}\text { Bipolar/ } \\
\text { LDO Positive } \\
\text { Voltage Regulator 5V }\end{array}$} & \multirow{4}{*}{ DC } & \multirow{4}{*}{$\begin{array}{l}\text { Exhibits LDR enhancement for functional } \\
\text { failures. Degradation level shows initial } \\
\text { failure dose levels. }\end{array}$} & \multirow{4}{*}{ G } & 0.01 & $50<V_{\text {out }}<60$ \\
\hline & & & & & & & 0.005 & $35<V_{\text {out }}<40$ \\
\hline & & & & & & & 0.001 & $10<V_{\text {out }}<15$ \\
\hline & & & & & & & 0.0005 & $7.5<V_{\text {out }}<10$ \\
\hline \multirow{3}{*}{$\begin{array}{l}\text { TL750M05CKTRR } \\
\text { (TO263-3) }\end{array}$} & \multirow{3}{*}{$\begin{array}{c}\text { Texas } \\
\text { Instruments }\end{array}$} & \multirow{3}{*}{0707} & \multirow{3}{*}{$\begin{array}{c}\text { Bipolar / } \\
\text { LDO positive } \\
\text { Voltage Regulator } 5 \mathrm{~V}\end{array}$} & \multirow{3}{*}{ DC } & \multirow{3}{*}{$\begin{array}{l}\mathrm{V}_{\text {out }} \text { failure levels }\left(\mathrm{I}_{\mathrm{O}}=10 \mathrm{~mA}\right) \\
5 \mathrm{mrad}(\mathrm{Si}) / \mathrm{s}: 70<\mathrm{V}_{\text {out }}<80 \mathrm{krad}(\mathrm{Si}) \\
1 \mathrm{mrad}(\mathrm{Si}) / \mathrm{s}:>20 \mathrm{krad}(\mathrm{Si}) \\
0.5 \mathrm{mrad}(\mathrm{Si}) / \mathrm{s}:>15 \mathrm{krad}(\mathrm{Si})\end{array}$} & \multirow{3}{*}{ G } & 0.005 & $70<V_{\text {out }}<80$ \\
\hline & & & & & & & 0.001 & $>20$ \\
\hline & & & & & & & 0.0005 & $>15$ \\
\hline \multirow{3}{*}{$\begin{array}{l}\text { LM1 17HRQMLV } \\
\text { (TO-39 metal can) }\end{array}$} & \multirow{3}{*}{ Natl Semi } & & & & LDR enhancement observed for $V_{\text {ref }}$ & & 0.005 & $>90$ \\
\hline & & 7D5867L019 & $\begin{array}{l}\text { Bipolar / Adjustable } \\
\text { Voltage Regulator }\end{array}$ & DC & Parameters within spec after $90,20, \& 15$ & G & 0.001 & $>20$ \\
\hline & & & & & $\mathrm{krad}(\mathrm{Si})$ for the $5,1, \& 0.5 \mathrm{mrad}(\mathrm{Si}) / \mathrm{s}$ parts. & & 0.0005 & $>15$ \\
\hline LM136AH2.5QMLV & Natl Semi & $200746 \mathrm{~K} 019$ & Bipolar I & DC & Exhibits no dose rate enhancement. & $\mathrm{G}$ & 0.005 & $>100$ \\
\hline
\end{tabular}

To be presented by Alvin Boutte at the International Electrical and Electronics Engineering (IEEE) Nuclear and Space Radiation Effects Conference (NSREC), July 27, 2011, Las Vegas, NV, and published on nepp.nasa.gov and radhome.gsfc.nasa.gov. 


\begin{tabular}{|c|c|c|c|c|c|c|c|c|}
\hline Part Number & Manufacturer & LDC & $\begin{array}{c}\text { Technology/ } \\
\text { Device Function }\end{array}$ & PI & Summary of Results & .气. & 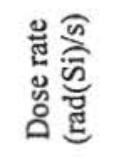 & 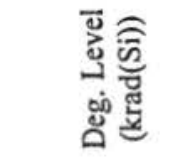 \\
\hline \multirow[t]{2}{*}{ (3-lead TO-46) } & & & \multirow[t]{2}{*}{ 2.5V Reference } & & \multirow{2}{*}{\begin{tabular}{|l|} 
Parameters w/in spec after $100,20, \& 10$ \\
$\operatorname{krad}(\mathrm{Si})$ for the $5,1, \& 0.5 \mathrm{mrad}(\mathrm{Si})$ devices.
\end{tabular}} & & 0.001 & $>20$ \\
\hline & & & & & & & 0.0005 & $>10$ \\
\hline \multirow{3}{*}{$\begin{array}{l}\text { RH1021CMW-5 } \\
\text { (Flatpack) }\end{array}$} & \multirow{3}{*}{$\begin{array}{c}\text { Linear } \\
\text { Technology }\end{array}$} & \multirow{3}{*}{$0123 \mathrm{~A}$} & \multirow{3}{*}{$\begin{array}{c}\text { Bipolar / Precision 5V } \\
\text { Reference }\end{array}$} & \multirow{3}{*}{$\mathrm{DC}$} & \multirow{3}{*}{$\begin{array}{l}\text { Parameters within spec after } 100,20, \& 10 \\
\operatorname{krad}(\mathrm{Si}) \text { for } 5,1, \& 0.5 \mathrm{mrad}(\mathrm{Si}) / \mathrm{s} .\end{array}$} & \multirow{3}{*}{ G } & 0.005 & $>100$ \\
\hline & & & & & & & 0.001 & $>20$ \\
\hline & & & & & & & 0.0005 & $>10$ \\
\hline \multirow{4}{*}{$\begin{array}{l}\text { RH1021CMH-5 } \\
\text { (TO-5 can) }\end{array}$} & \multirow{4}{*}{$\begin{array}{c}\text { Linear } \\
\text { Technology }\end{array}$} & \multirow{4}{*}{$9783 \mathrm{~A}$} & \multirow{4}{*}{$\begin{array}{l}\text { Bipolar/ } \\
\text { Precision 5V } \\
\text { Reference }\end{array}$} & \multirow{4}{*}{ DC } & \multirow{4}{*}{$\begin{array}{l}\text { LDR enhancement observed for parts } \\
\text { irradiated at } 5 \mathrm{mrad}(\mathrm{Si}) / \mathrm{s} \text { after } 30 \mathrm{krad}(\mathrm{Si}) .5 \\
\text { mrad(Si) }(\mathrm{TO}-5): 90<\mathrm{V}_{\mathrm{z}}<100 \mathrm{krad}(\mathrm{Si}) .\end{array}$} & \multirow{4}{*}{ G } & 0.01 & $>50$ \\
\hline & & & & & & & 0.005 & $80<V_{\mathrm{z}}<90$ \\
\hline & & & & & & & 0.001 & $>20$ \\
\hline & & & & & & & 0.0005 & $>10$ \\
\hline \multirow{3}{*}{$\begin{array}{l}\text { RH1009MW } \\
\text { (Flatpack) }\end{array}$} & \multirow{3}{*}{$\begin{array}{c}\text { Linear } \\
\text { Technology }\end{array}$} & \multirow{3}{*}{$0649 \mathrm{~A}$} & \multirow{3}{*}{$\begin{array}{c}\text { Bipolar / } \\
\text { 2.5V Reference }\end{array}$} & \multirow{3}{*}{ DC } & \multirow{3}{*}{$\begin{array}{l}\text { Exhibits dose rate enhancement after } 15 \\
\text { krad(Si) for devices irradiated at } 5 \& 1 \\
\operatorname{mrad}(\mathrm{Si}) / \mathrm{s} .5 \mathrm{mrad}(\mathrm{Si}) / \mathrm{s} \text { Flatpacks: } 100<\mathrm{V}_{\mathrm{z}} \\
<120 \mathrm{krad}(\mathrm{Si}) \text {. }\end{array}$} & \multirow{3}{*}{ G } & 0.005 & $100<\mathrm{V}_{2}<120$ \\
\hline & & & & & & & 0.001 & $>20$ \\
\hline & & & & & & & 0.0005 & $>10$ \\
\hline \multirow{3}{*}{$\begin{array}{l}\text { RH1009MH } \\
\text { (TO-46 can) }\end{array}$} & \multirow{3}{*}{$\begin{array}{c}\text { Linear } \\
\text { Technology }\end{array}$} & \multirow{3}{*}{$0829 \mathrm{H}$} & \multirow{3}{*}{$\begin{array}{l}\text { Bipolar / } \\
\text { 2.5V Reference }\end{array}$} & \multirow{3}{*}{ DC } & \multirow{3}{*}{$\begin{array}{l}\text { Exhibits dose rate enhancement after } 20 \\
\mathrm{krad}(\mathrm{Si}) \text { for devices irradiated at } 5 \text { \& } 1 \\
\mathrm{mrad}(\mathrm{Si}) / \mathrm{s} .5 \mathrm{mrad}(\mathrm{Si}) / \mathrm{s} \text { TO-46 cans: } 80<\mathrm{V}_{2} \\
<90 \mathrm{krad}(\mathrm{Si}) \text {. }\end{array}$} & \multirow{3}{*}{ G } & 0.005 & $80<V_{z}<90$ \\
\hline & & & & & & & 0.001 & $>20$ \\
\hline & & & & & & & 0.0005 & $>10$ \\
\hline \multirow{3}{*}{$\begin{array}{l}\text { RHFL4913ESY332 } \\
\text { (TO257) }\end{array}$} & \multirow{3}{*}{$\begin{array}{c}\text { ST } \\
\text { Micro- } \\
\text { electronics }\end{array}$} & 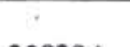 & Bipolar / & & Parameters within spec for parts irradiated at & & 0.001 & $>100$ \\
\hline & & & Voltage Regulator & DC & $10,5 \& 1 \mathrm{mrad}(\mathrm{Si}) / \mathrm{s}$ after $100,30 \& 20$ & G & 0.005 & $>30$ \\
\hline & & & & & $\operatorname{krad}(\mathrm{Si})$ & & 0.001 & $>20$ \\
\hline RHFL4913KP332 & & & Bipolar / & & Parameters within spec for parts irradiated at & & 0.01 & $>100$ \\
\hline (Flat-16) & $\begin{array}{l}\text { Micro- } \\
\text { electronics }\end{array}$ & 30814B & Voltage Regulator & DC & $10,5 \& 1 \mathrm{mrad}(\mathrm{Si}) / \mathrm{s}$ after $100,30 \& 20$ & G & 0.005 & $>30$ \\
\hline RHF43B (Ceramic Flat-8) & $\begin{array}{c}\text { ST } \\
\text { Micro- } \\
\text { electronics }\end{array}$ & $30820 \mathrm{~A}$ & $\begin{array}{c}\text { Bipolar/ } \\
\text { Voltage Regulator }\end{array}$ & DC & $\begin{array}{l}\text { Parameters within spec for parts irradiated at } \\
0.5 \mathrm{mrad}(\mathrm{Si}) / \mathrm{s} \text { after } 10 \mathrm{krad}(\mathrm{Si}) .\end{array}$ & G & 0.0005 & $>10$ \\
\hline RHI02I & $\begin{array}{c}\text { Linear } \\
\text { Technology }\end{array}$ & 0940A & $\begin{array}{c}\text { Bipolar/ } \\
\text { 5V Voltage Reference }\end{array}$ & $\mathrm{MeC}$ & $\begin{array}{l}\mathrm{V}_{\text {out }} \text { exceeded the specified limits between } 10 \\
\& 30 \mathrm{krad}(\mathrm{Si}) .\end{array}$ & G & 55.6 & $10<V_{\text {out }}<30$ \\
\hline
\end{tabular}

To be presented by Alvin Boutte at the International Electrical and Electronics Engineering (IEEE) Nuclear and Space Radiation Effects Conference (NSREC), July 27, 2011, Las Vegas, NV, and published on nepp.nasa.gov and radhome.gsfc.nasa.gov. 


\section{Displacement Damage}

\begin{tabular}{|c|c|c|c|c|c|c|c|c|}
\hline Part Number & Manufacturer & LDC & $\begin{array}{c}\text { Technology/ } \\
\text { Device Function }\end{array}$ & PI & Summary of Results & 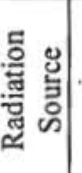 & 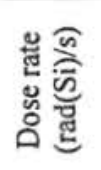 & 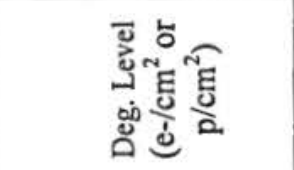 \\
\hline 66212 & Micropac ${ }^{\circ}$ & 1014 & GaAIAs/Optocoupler & JP & $\begin{array}{l}\text { Parts remained functional through } 2 \mathrm{e}^{12} \mathrm{~cm}^{2} \text {. } \\
\text { The device CTR dropped out of datasheet } \\
\text { specifications between } 4 \times 10^{11} \mathrm{~cm}^{-2} \& 8 \times 10^{11} \\
\mathrm{~cm}^{-2} \text { at a } \mathrm{V}_{\mathrm{ce}} \text { of } 1 \mathrm{~V} \& \text { between } 8 \times 10^{11} \mathrm{~cm}^{-2} \\
\& 1.2 \times 10^{12} \mathrm{~cm}^{-2} \text { at a } \mathrm{V}_{\mathrm{ce}} \text { of } 5 \mathrm{~V} \text {. Tested with } \\
50 \mathrm{MeV} \text { protons. }\end{array}$ & $P$ & N/A & $\begin{array}{c}4 \times 10^{11} \leq \mathrm{x} \leq 8 \times 10^{11} \\
\left(\mathrm{~V}_{\mathrm{CE}}=1 \mathrm{~V}\right) \\
8 \times 10^{11} \leq \mathrm{x} \leq 1.2 \times 10^{12} \\
\left(\mathrm{~V}_{\mathrm{CE}}=5 \mathrm{~V}\right)\end{array}$ \\
\hline HCPL-573K & Avago & 0937 & GaAsP/Optocoupler & JP & $\begin{array}{l}\text { Parts remained functional through } 2 \times 10^{12} \\
\mathrm{~cm}^{-2} \text {. CTR within spec at a } \mathrm{V}_{\mathrm{ce}} \text { of } 0.4 \mathrm{~V} \text { for } \\
\text { all fluences tested. CTR out of specification } \\
\text { at a } \mathrm{V}_{\mathrm{ce}} \text { of } 0.1 \mathrm{~V} \text { at } 2 \times 10^{12} \mathrm{~cm}^{-2} \text {. Tested with } \\
50 \mathrm{MeV} \text { protons. }\end{array}$ & $P$ & N/A & $\begin{array}{c}1.2 \times 10^{12} \leq \mathrm{x} \leq 2 \times 10^{12} \\
\left(\mathrm{~V}_{\mathrm{CE}}=0.1 \mathrm{~V}\right) \\
>2 \times 10^{12} \\
\left(\mathrm{~V}_{\mathrm{CE}}=0.4 \mathrm{~V}\right)\end{array}$ \\
\hline RAM-3+ & Mini-Circuits & 0918 & InGaP/MMIC RF Amp & JP & $\begin{array}{l}\text { Parts remained functional \& in spec through } \\
2 \times 10^{12} \mathrm{~cm}^{-2} \text {. Mini-Circuits evaluation board } \\
\text { used for testing. Tested with } 50 \mathrm{MeV} \\
\text { protons. }\end{array}$ & $\mathbf{P}$ & N/A & $>2 \times 10^{12}$ \\
\hline UT54LVDS031LV/E & Aeroflex & 0946 & $\begin{array}{c}\text { CMOS / Low Voltage } \\
\text { Quad Driver }\end{array}$ & $\mathrm{MeC}$ & $\begin{array}{l}\text { Within the measurement resolution \& noise } \\
\text { threshold, all parameters stayed in spec to a } \\
17 \mathrm{MeV} \text { electron fluence of } 8 \times 10^{13} \mathrm{~cm}^{-2} \text {. }\end{array}$ & E & N/A & $>8 \times 10^{13}$ \\
\hline MCM2760-4M & QTech & 0135 & $\begin{array}{l}\text { Hybrid / Crystal } \\
\text { Oscillator }\end{array}$ & $\mathrm{MeC}$ & $\begin{array}{l}\text { All parameters stayed within spec limits to a } \\
17 \mathrm{MeV} \text { electron fluence of } 1.5 \times 10^{14} \mathrm{~cm}^{-2} \text {. }\end{array}$ & E & N/A & $>1.5 \times 10^{14}$ \\
\hline UT54ACS08 & Aeroflex & 0907 & $\begin{array}{l}\text { CMOS / Radiation- } \\
\text { Hardened Quadruple } \\
\text { 2-Input AND Gates }\end{array}$ & $\mathrm{MeC}$ & $\begin{array}{l}\mathrm{V}_{\mathrm{IH}} \text { decreased below the spec between } 17 \\
\mathrm{MeV} \text { electron fluences of } 8 \times 10^{13} \text { and } 1.6 \times \\
10^{14} \mathrm{~cm}^{-2} \text {. }\end{array}$ & E & N/A & $8 \times 10^{13} \leq x \leq 1.6 \times 10^{14}$ \\
\hline LS2805 & $\begin{array}{c}\text { Intl } \\
\text { Rectifier }\end{array}$ & 0536 & $\begin{array}{c}\text { Hybrid /High } \\
\text { Reliability Radiation } \\
\text { Hardened DC/DC } \\
\text { Converter }\end{array}$ & $\mathrm{MeC}$ & $\begin{array}{l}\mathrm{V}_{\text {out }} \text { decreased below the specified limit } \\
\text { between } 17 \mathrm{MeV} \text { electron fluences of } 2.7 \text { and } \\
5.3 \times 10^{13} \mathrm{~cm}^{-2} \text {. }\end{array}$ & $\mathrm{E}$ & N/A & $\begin{array}{l}2.7 \times 10^{13} \leq x \leq \\
5.3 \times 10^{13}\end{array}$ \\
\hline 2 N5116 & Vishay & S0618 & $\begin{array}{l}\text { JFET / P-Channel } \\
\text { Analog Switch }\end{array}$ & $\mathrm{MeC}$ & $\begin{array}{l}\text { Within the measurement resolution \& noise } \\
\text { threshold, all parameters stayed in spec to a } \\
17 \mathrm{MeV} \text { electron fluence of } 2.7 \times 10^{14} \mathrm{~cm}^{-2} \text {. }\end{array}$ & $\mathrm{E}$ & N/A & $>2.7 \times 10^{14}$ \\
\hline \multirow{2}{*}{$2 \mathrm{~N} 2222$} & \multirow{2}{*}{ Semicoa } & \multirow{2}{*}{0743} & \multirow{2}{*}{$\begin{array}{c}\text { Bipolar / Silicon NPN } \\
\text { Transistor }\end{array}$} & \multirow{2}{*}{$\mathrm{MeC}$} & $\begin{array}{l}\mathrm{h}_{\mathrm{FE}} \text { for } \mathrm{I}_{\mathrm{C}} \text { of } 0.1,1.0, \& 10 \mathrm{~mA} \text { exceeded the } \\
\text { spec between } 5 \mathrm{MeV} \text { electron fluences of } 1.1 \\
\text { and } 3.6 \times 10^{13} \mathrm{~cm}^{-2} \text { for all three parts. }\end{array}$ & E & N/A & $\begin{array}{l}1.1 \times 10^{13} \leq x \leq \\
3.6 \times 10^{13}\end{array}$ \\
\hline & & & & & $\begin{array}{l}\mathrm{h}_{\mathrm{FE}} \text { for } \mathrm{I}_{\mathrm{C}} \text { of } 0.1,1.0, \& 10 \mathrm{~mA} \text { exceeded the } \\
\text { spec between } 17 \mathrm{MeV} \text { electron fluences of } 8 \\
\times \mathrm{I} 0^{12} \text { and } 2.7 \times 10^{13} \mathrm{~cm}^{-2} \text { for all three parts. }\end{array}$ & E & N/A & $8 \times 10^{12} \leq x \leq 2.7 \times 10^{13}$ \\
\hline
\end{tabular}

To be presented by Alvin Boutte at the International Electrical and Electronics Engineering (IEEE) Nuclear and Space Radiation Effects Conference (NSREC), July 27, 2011, Las Vegas, NV, and published on nepp.nasa.gov and radhome.gsfc.nasa.gov. 


\begin{tabular}{|c|c|c|c|c|c|c|c|c|c|}
\hline Part Number & & Manufacturer & LDC & $\begin{array}{l}\text { Technology/ } \\
\text { Device Function }\end{array}$ & PI & Summary of Results & 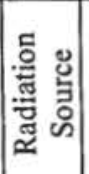 & 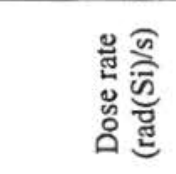 & 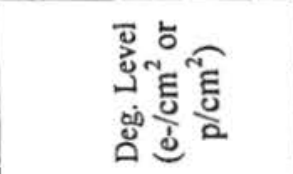 \\
\hline & & & & & & $\begin{array}{l}\mathrm{h}_{\mathrm{FE}} \text { for } \mathrm{I}_{\mathrm{C}} \text { of } 0.1,1.0, \& 10 \mathrm{~mA} \text { exceeded the } \\
\text { spec between } 25 \mathrm{MeV} \text { electron fluences of } 7 \\
\mathrm{x} 10^{12} \text { and } 2.3 \times 10^{13} \mathrm{~cm}^{-2} \text { for all three parts. }\end{array}$ & $\mathrm{E}$ & N/A & $7 \times 10^{12} \leq x \leq 2.3 \times 10^{13}$ \\
\hline $2 \mathrm{~N} 2907$ & & Micro Semi & 0513 & $\begin{array}{l}\text { Bipolar / PNP Silicon } \\
\text { Switching Transistor }\end{array}$ & $\mathrm{MeC}$ & $\begin{array}{l}h_{\mathrm{FE}} \text { for a } \mathrm{I}_{\mathrm{C}} \text { of } 0.1 \mathrm{~mA} \text { exceeded the spec at a } \\
17 \mathrm{MeV} \text { electron fluence of } 2.7 \times 10^{11} \mathrm{~cm}^{-2} \text {, } \\
\text { the first irradiation step. }\end{array}$ & E & N/A & $0 \leq x \leq 2.7 \times 10^{11}$ \\
\hline RH1021 & & $\begin{array}{l}\text { Linear } \\
\text { Technology }\end{array}$ & 0940A & $\begin{array}{l}\text { Bipolar / 5V Voltage } \\
\text { Reference }\end{array}$ & $\mathrm{MeC}$ & $\begin{array}{l}V_{\text {out }} \text { exceeded the specified limits at a } 17 \\
\text { MeV electron fluence of } 2.7 \times 10^{11} \mathrm{~cm}^{-2} \text {, the } \\
\text { first irradiation step. }\end{array}$ & $\mathrm{E}$ & N/A & $0 \leq x \leq 2.7 \times 10^{11}$ \\
\hline SPT6235MS & & SSDI & 0624 & $\begin{array}{l}\text { General Purpose High } \\
\text { Voltage/Power } \\
\text { NPN BJT }\end{array}$ & JP & $\begin{array}{l}\text { Tested with } \mathrm{I}_{\mathrm{c}} \text { of } 0.25 \text { \& } 0.51 \mathrm{~A}, \mathrm{~V}_{\mathrm{b}}=5 \mathrm{~V}, \& \\
\mathrm{~V}_{\mathrm{ce}}=155.6 \mathrm{~V} \text {. Mean } \mathrm{h}_{\mathrm{FE}} \text { stayed within } \\
\text { application spec for the duration of the } 17 \\
\text { MeV electron exposures. Biased parts were } \\
\text { tested to an electron fluence of } 3.3 \times 10^{13} \mathrm{~cm}^{-2} \\
\text { and the unbiased parts to } 7.1 \times 10^{13} \mathrm{~cm}^{-2} \text {. }\end{array}$ & $\mathrm{E}$ & N/A & $\begin{array}{c}>3.3 \times 10^{13} \text { (biased) } \\
>7.1 \times 10^{13} \text { (unbiased) }\end{array}$ \\
\hline JANS2N3866 & . & Semicoa & 0721 & $\begin{array}{l}\text { General Purpose } \\
\text { NPN BJT }\end{array}$ & JP & $\begin{array}{l}\text { Tested with } \mathrm{I}_{\mathrm{c}} \text { of } 0.25 \& 0.51 \mathrm{~A} \text { with a pulsed } \\
\text { base current \& } \mathrm{V}_{\mathrm{ce}}=4.4 \mathrm{~V} \text {. Mean } \mathrm{h}_{\mathrm{FE}} \text { stayed } \\
\text { within application spec for the duration of } \\
\text { the } 17 \mathrm{MeV} \text { electron exposures. Biased parts } \\
\text { were tested to an electron fluence of } 3.3 \times 10^{13} \\
\mathrm{~cm}^{-2} \text { and the unbiased parts to } 7.1 \times 10^{13} \mathrm{~cm}^{-2} \text {. }\end{array}$ & E & N/A & $\begin{array}{c}>3.3 \times 10^{13} \text { (biased) } \\
>7.1 \times 10^{13} \text { (unbiased) }\end{array}$ \\
\hline
\end{tabular}




\section{Test Results and Discussion}

As in our past workshop compendia of GSFC test results, each DUT has a detailed test report available online at http://radhome.gsfc.nasa.gov [3] describing in further detail, test method, TID conditions/parameters, test results, and graphs of data.

\section{A. 66212/Optocoupler (Displacement Damage)/Micropac}

We tested five pieces of the $66212850 \mathrm{~nm}$ optocoupler from Micropac Industries, Inc. for DD dose degradation using the accelerated proton beam at the LBNL BASE facility during June 2010. The CTR of the amplifier was monitored as a function of proton fluence by measuring the $I_{C E}$ as a function of the $I_{F}$. The parts were irradiated under bias.

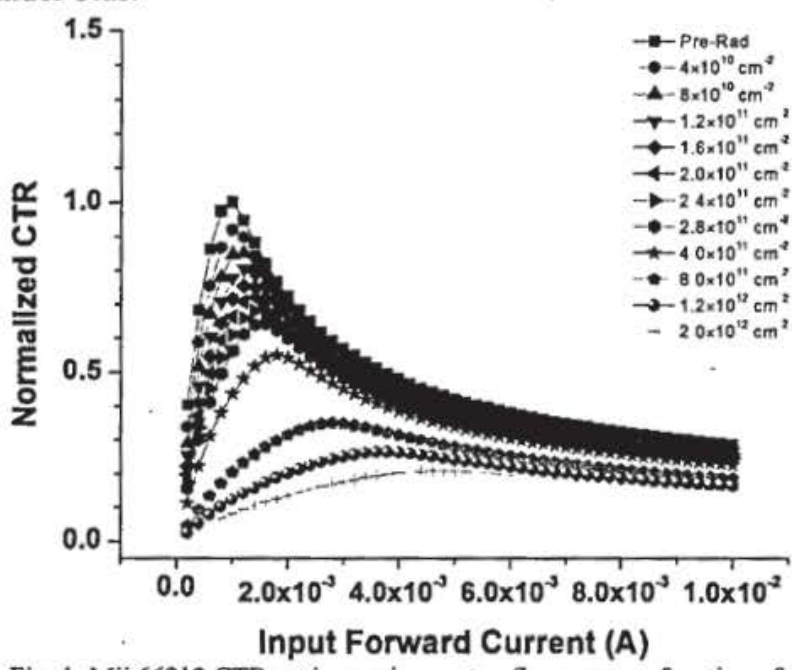

Fig. 1. Mii 66212 CTRs at increasing proton fluences as a function of input forward current. The CTRs shown here are an average of all the pieces tested. For all data, $\mathrm{V}_{\mathrm{CE}}=1 \mathrm{~V}$.

The 66212 optocoupler consists of an $850 \mathrm{~nm}$ GaAlAs LED optically coupled to a phototransistor detector all mounted in a hermetic 4-pin LCC package. Following irradiation to $2 \times 10^{12} \mathrm{p} / \mathrm{cm}^{2}$ with $50 \mathrm{MeV}$ protons, degradation was observed in all devices tested. 99/90 bounds were computed based on the sample standard deviation and one-sided tolerance limits. The device CTR dropped out of datasheet specifications between $4 \times 10^{11}$ $\mathrm{cm}^{-2}$ and $8 \times 10^{11} \mathrm{~cm}^{-2}$ at a $V_{\mathrm{CE}}$ of $1 \mathrm{~V}$. The measured CTR's at a $V_{\text {CE }}$ of $1 \mathrm{~V}$ are shown in Fig. 1. The CTR was normalized to the peak average CTR pre-irradiation; at a $V_{\text {CE }}$ of $1 \mathrm{~V}$ this was 8.9 .

\section{B. AD648/Operational Amplifier/Analog Devices}

The AD648 is a pair of low-power, precision op amps with JFET inputs manufactured by Analog Devices. The parts were tested for ELDRS using a Co-60 source, at a dose rate of $10 \mathrm{mrad} / \mathrm{s}$ to a total dose of $20 \mathrm{krad}(\mathrm{Si})$. A total of ten parts (20 op amps) were irradiated, with five parts irradiated under biased and five parts with all pins grounded, and an additional two parts were used as controls. Fig. 2 shows the average Ib for the control samples, the parts biased during irradiation, and the parts with all pins grounded during irradiation as a function of dose. At the $10 \mathrm{krad}(\mathrm{Si})$ dose, all biased parts exceeded the specification of $\mathrm{I}_{\text {bias }}$ and $\mathrm{I}_{\mathrm{os}}$, but some parts exceeded the specifications as early as $2 \mathrm{krad}(\mathrm{Si})$. In the case of the unbiased parts, the average $I_{\text {bias }}$ also exceeded the specification for $I_{\text {bias }}$ at $10 \mathrm{krad}(\mathrm{Si})$, but all other parameters remained within specification to the 20 $\operatorname{krad}(\mathrm{Si}) \operatorname{dose}$.

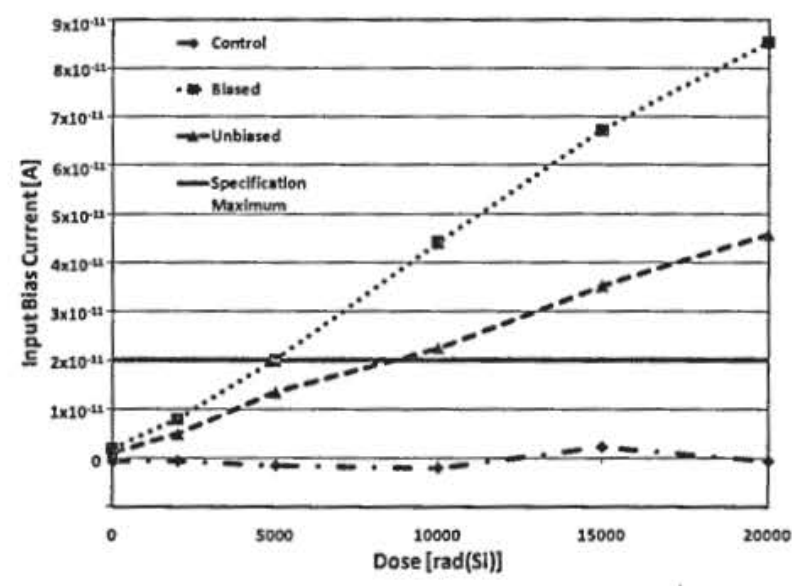

Fig. 2. The average $I_{\text {bias }}$ for the AD648 as a function of dose.

\section{C.AD5544/Digital to Analog Converter/Analog Devices}

The AD5544 is a quad, 16-bit, current-output digital-toanalog converter manufactured by Analog Devices. Until mid-2008, the device was manufactured in a BiCMOS technology, while after this date the device was migrated to a pure CMOS technology. Testing was carried out for parts in both technologies. The most notable characteristic of the initial tests done on CMOS technology (LDC 0827) was part-to-part variability; one part failed below $2 \mathrm{krad}(\mathrm{Si})(\mathrm{DNL})$ and some parts performed well up to the highest test dose of $50 \mathrm{krad}(\mathrm{Si})$. Moreover, multiple failure modes in DNL were noted for different parts and no obvious correlation was seen for failures in the two sensitive parameters-see Figs. 3 and 4 . While not a source of component failure in the intended application, IDD was also sensitive to dose, rising rapidly in the biased parts above $15 \mathrm{krad}(\mathrm{Si})$ and reaching more than $10 \mathrm{~mA}$ by $50 \mathrm{krad}(\mathrm{Si})$. IDD was unaffected in the grounded components.

Because of the anomalous nature of the results, an additional 5 parts from date code 0827 were tested; along with 5 parts from another CMOS lot, date code 1028 . Because the biased parts in previous testing yielded worstcase results, the second batch of parts was irradiated under bias. The concern here was to ensure that the previous results were not due to a bad lot, mishandling, or electrostatic discharge. These tests yielded two additional failures. One part from date code 1028 failed between 7 and $10 \mathrm{krad}(\mathrm{Si})$, and one part from date code 0827 failed between 20 and $30 \mathrm{krad}(\mathrm{Si})$. 
At this stage, the project halted qualification of the CMOS AD5544s and procured older BiCMOS parts (date codes 0332 and 0409). Testing for these parts has been completed but further analysis is underway.

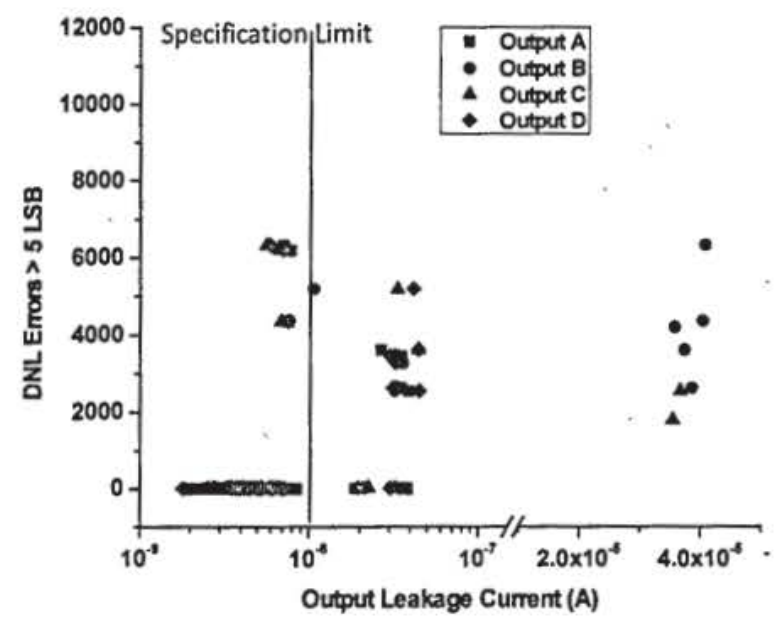

Fig. 3. Biased DNL errors versus output leakage current for all four DACs (A, B, C, \& D). LDC 0827 - CMOS Technology.

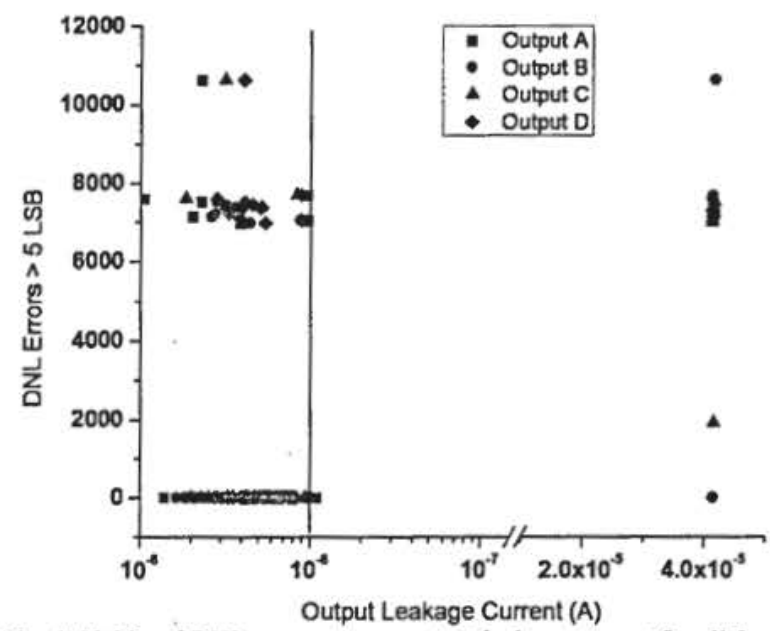

Fig. 4. Unbiased DNL errors versus output leakage current for all four DACs (A, B, C, \& D). LDC 0827 - CMOS Technology.

\section{SUMMARY}

We have presented data from recent TID and protoninduced damage tests on a variety of primarily commercial devices. It is the authors' recommendation that this data be used with caution due to many application/lot-specific issues. We also highly recommend that lot testing be performed on any suspect or commercial device.

\section{Acknowledgment}

The Authors would like to acknowledge the sponsors of this effort: NASA Electronic Parts and Packaging Program (NEPP), NASA Flight Projects, and the Defense Threat Reduction Agency (DTRA) under IACRO\# 10-4977I and 11-43951. The authors thank members of the Radiation
Effects and Analysis Group (REAG) who contributed to the test results presented here, Melanie D. Berg, Mark Friendlich, Hak S. Kim, Anthony M. Dung-Phan, Donald K. Hawkins, James D. Forney, Tim Irwin, Christina M. Seidleck, and Stephen R. Cox.

\section{REFERENCES}

[1] Martha V. O'Bryan, et al., "Recent Single Event Effects Compendium of Candidate Electronics for NASA Space Systems," to be submitted for presentation at IEEE NSREC 2011 Radiation Effects Data Workshop, July 2011.

[2] Department of Defense "Test Method Standard Microcircuits," MIL-STD-883 Test Method 1019.8 lonizing radiation (total dose) test procedure, September 30, 2010, http://www.dscc.dla.mil/Downloads/MilSpec/Docs/MIL-STD883/std883_1000.pdf. NASA/GSFC Radiation Effects and Analysis home page, http://radhome.gsfc.nasa.gov.

[3] NASA Electronic Parts and Packaging Program home page, http://nepp.nasa.gov. 